\title{
The long and winding route
}

\author{
James I. Fann, MD
}

From the Department of Cardiothoracic Surgery, Stanford University, Stanford, Calif

Disclosures: Author has nothing to disclose with regard to commercial support.

Received for publication Dec 4, 2017; accepted for publication Dec 14, 2017; available ahead of print Jan 10, 2018 .

Address for reprints: James I. Fann, MD, Department of Cardiothoracic Surgery, Stanford University, 300 Pasteur Drive, Stanford, CA 94305 (E-mail: jfann@stanford.edu).

J Thorac Cardiovasc Surg 2018;155:1578-9

0022-5223/\$00.00

Published by Elsevier Inc. on behalf of The American Association for Thoracic Surgery https://doi.org/10.1016/j.jtcvs.2017.12.059

No one would contend with the notion that innovative endovascular surgery for abdominal aortic aneurysmal and occlusive disease has forever changed our management of these disorders. In terms of morbidity and mortality, less invasive in these cases can lead to better outcomes. Therefore, when one proposes the idea of increasing the invasiveness of the surgical approach for aortoiliac occlusive disease, a little dose of circumspection is in order. With a long and respectable history, the lesser operation of the extra-anatomical axillofemoral bypass graft remains in the surgical armamentarium in treating those with marked comorbidities and anatomic challenges in the abdomen. Albeit with lower patency rates than what one would consider to be ideal, such an approach has served many reasonably well over the years.

In this issue of the Journal, Bosse and colleagues ${ }^{1}$ reintroduce the concept of central aortic inflow for an extraanatomical "ventral aorta" course of bypass graft in patients with severe aortoiliac occlusive disease. Although this technique has been used in the past, the distinction is that the patients in this study are those who require concomitant (and primarily) cardiac surgery. To accept the authors' thesis, one must first confirm that patients with coexisting symptomatic aortoiliac occlusion are not amenable to endovascular or lesser invasive procedures in the setting of serious comorbidities and anatomic barriers. Second, the advantages of a central aortic inflow (recognizing the risk-benefit ratio) compared with a lesser artery as the source of inflow for an extra-anatomic bypass have to be defined. Further and very importantly, whether a lowerextremity revascularization procedure needs to be performed at the time of open cardiac surgery as opposed to sequentially at some point after recovery should be medically justified. Thus, thoughtful decision-making and careful patient selection are imperative if one were to consider a concomitant surgical approach in this limited patient population.

Notwithstanding, the argument for the "ventral aorta" gets a boost when one considers that since the ascending aorta will be exposed with the concurrent cardiac surgery

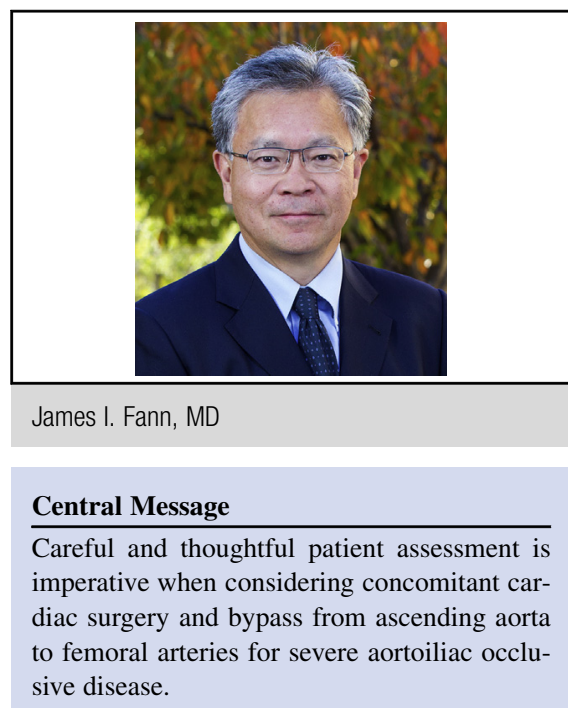

See Article page 1574.

(in this case, coronary artery bypass grafting, valve surgery, or innominate artery surgery), perhaps there is a lower threshold of acceptance for a central aortic origin of the graft to the femoral arteries, especially if the course of the graft is well mapped out and not overly traumatic. This long and relatively straight preperitoneal route, staying anterior to the posterior rectus fascia and above the inguinal ligament, is associated with a potentially winding portion at the femoral region with Y-configuration to the 2 limbs (or bifemoral components) of the graft. But again, the reality checker dictates that this lower-extremity revascularization procedure should be considered only if it does not pose significant added stress or risk to the patients at the time of cardiac surgery.

Recognizing the many caveats and potential pitfalls, Bosse and colleagues ${ }^{1}$ report a small but impressive series of 8 patients who have benefited from the combined cardiac and lower-extremity revascularization approach. No perioperative deaths or major complications related to the "ventral aorta" procedure occurred. At 5 years, but with only a median follow-up of 11 months, 1 patient developed graft thrombosis of 1 limb of the graft at 9 months after surgery; also, 1 patient required a femoral endarterectomy at 4 years, not an unexpected event in terms of disease progression in this population. The authors conclude that the absence of major postoperative complications and the acceptable reported patency rate illustrate the positive aspects of a combined strategy. 
So, in these patients with severe cardiac disease and aortoiliac occlusive disease, the latter of which cannot be safely treated with an endovascular or open abdominal procedure or optimally managed with a sequential approach, combined cardiac surgery and an extraanatomic bypass grafting using the ascending aorta as inflow can be considered. Again, whether such a bypass procedure should be performed at the same time as the open cardiac procedure mandates careful and thoughtful patient assessment. Even with more robust inflow from the ascending aorta, the long and potentially winding route of the "ventral aorta" to femoral bypass graft will require long-term patency and, perhaps more importantly, long-term survival data to demonstrate overall patient benefit.

\section{Reference}

1. Bosse C, Ramadan R, Fabre D, Guihaire J. Cardiac surgery combined with bypass from the ascending aorta to the bilateral femoral arteries for severe aorto-iliac occlusion: a case series. J Thorac Cardiovasc Surg. 2018;155:1574-7. 